\title{
Mesial temporal lobe epilepsy with childhood febrile seizure.
}

\author{
Ali Akbar Asadi-Pooya \\ Thomas Jefferson University \\ Maromi Nei \\ Thomas Jefferson University \\ Cyrus Rostami \\ Thomas Jefferson University \\ Michael R. Sperling \\ Thomas Jefferson University
}

Follow this and additional works at: https://jdc.jefferson.edu/neurologyfp

Part of the Neurology Commons

Let us know how access to this document benefits you

\section{Recommended Citation}

Asadi-Pooya, Ali Akbar; Nei, Maromi; Rostami, Cyrus; and Sperling, Michael R., "Mesial temporal lobe epilepsy with childhood febrile seizure." (2016). Department of Neurology Faculty Papers.

Paper 113.

https://jdc.jefferson.edu/neurologyfp/113

This Article is brought to you for free and open access by the Jefferson Digital Commons. The Jefferson Digital Commons is a service of Thomas Jefferson University's Center for Teaching and Learning (CTL). The Commons is a showcase for Jefferson books and journals, peer-reviewed scholarly publications, unique historical collections from the University archives, and teaching tools. The Jefferson Digital Commons allows researchers and interested readers anywhere in the world to learn about and keep up to date with Jefferson scholarship. This article has been accepted for inclusion in Department of Neurology Faculty Papers by an authorized administrator of the Jefferson Digital Commons. For more information, please contact: JeffersonDigitalCommons@jefferson.edu. 
Title: Mesial temporal lobe epilepsy with childhood febrile seizure.

\section{Ali A. Asadi-Pooya, M.D., Maromi Nei, M.D., Cyrus Rostami, Michael R. Sperling, M.D.}

Jefferson Comprehensive Epilepsy Center, Department of Neurology, Thomas Jefferson

University, Philadelphia, Pennsylvania, U.S.A.

\section{Address for correspondence:}

Ali A. Asadi-Pooya, M.D.

Department of Neurology

900 Walnut Street, Suite 200

Philadelphia, PA 19107

Phone: 215-955-1222

Fax: 215-955-3745

E-mail: aliasadipooya@yahoo.com

Running title: Febrile seizure and mesial temporal sclerosis.

Key words: Mesial temporal sclerosis; Outcome; Surgery; Febrile seizure.

Word count for the paper: 1580. Word count for the abstract: 250 .

Number of references: 12 .

Number of tables: 1 .

Number of figures: 1 . 


\begin{abstract}
:
Objectives: To evaluate the demographic and clinical manifestations of patients with mesial temporal sclerosis and temporal lobe epilepsy (MTS-TLE) with childhood febrile seizure (FS) and establishing the potential differences as compared to those without FS. We also investigated the surgery outcome in these two groups of patients.
\end{abstract}

Materials \& methods: In this retrospective study all patients with a clinical diagnosis of drugresistant TLE due to mesial temporal sclerosis, who underwent epilepsy surgery at Jefferson comprehensive epilepsy center, were recruited. Patients were prospectively registered in a database from 1986 through 2014. Post-surgical outcome was classified into two groups; seizurefree or relapsed. Clinical manifestations and outcome were compared between patients with MTS-TLE with FS and those without FS.

Results: Two-hundred sixty-two patients were eligible for this study. One hundred seventy patients (64.9\%) did not have FS in their childhood, while 92 patients $(35.1 \%)$ reported experiencing FS in their childhood. Demographic and clinical characteristics of these two groups of patients were not different. Postoperative seizure outcome was not statistically different between these two groups of patients $(\mathrm{p}=0.19)$.

Conclusions: When MTS is the pathological substrate of TLE, clinical manifestations and response to surgical treatment of patients are very similar in patients with history of febrile seizure in their childhood compared to those without such an experience. In other words, when the subgroup of patients with MTS-TLE and drug resistant seizures is examined history of childhood febrile seizure loses its value as a distinguishing factor in characteristics or predictive factor for surgery outcome.

Key words: Mesial temporal sclerosis; Outcome; Surgery; Febrile seizure. 


\section{Introduction}

Mesial temporal sclerosis (MTS) is the most common pathological substrate of temporal lobe epilepsy (TLE) (1). Temporal lobe epilepsy with MTS is one of the most common types of drug-resistant epilepsy referred for epilepsy surgery; it is often refractory to antiepileptic drugs (AEDs), but responds favorably to surgery $(2,3)$. There is a relationship between febrile seizure (FS) in childhood and subsequent MTS, but this relationship is a controversial issue in the literature. In one study, MRI findings of a markedly hyperintense hippocampus in children with febrile status epilepticus was highly associated with subsequent mesial temporal sclerosis (4). However, there is also evidence that suggests a subtle, pre-existing hippocampal malformation may exist in some patients that may facilitate febrile convulsions and contribute to the development of subsequent MTS (5). The question whether TLE with childhood febrile seizures is a distinctive entity has been raised before in the literature $(6,7)$. However, the findings in two previous studies are contradictory. One study identified TLE-FS as a phenotype that can be delineated from other TLE (6), while another study concluded that medial TLE with complex febrile seizure is not a distinct epilepsy syndrome (7).

The aim of this study was to evaluate the demographic and clinical manifestations of MTS-TLE with FS in childhood and identify potential differences as compared with manifestations observed in MTS-TLE without FS in a large surgical cohort. We also investigated the surgery outcome in these two groups of patients. 


\section{Material and Methods}

In this retrospective study, all patients with a clinical diagnosis of drug resistant TLE due to mesial temporal sclerosis who underwent epilepsy surgery at Jefferson comprehensive epilepsy center were recruited. Patients were prospectively registered in a database from 1986 through 2014. The diagnosis of MTS-TLE was made by the epileptologists working at this institution based on clinical grounds (semiology), electroencephalographic (EEG) findings and imaging [magnetic resonance imaging (MRI)]. There was no age limit to enter the study. For all patients, a comprehensive presurgical evaluation including a 1.5-Tesla brain MRI (epilepsy protocol) was performed. Magnetic resonance imaging studies were analyzed by neuroradiologists, neurologists, and neurosurgeons with expertise in epilepsy. We classified patients as having MTS if they had clear signs of mesial temporal atrophy and/or sclerosis in their brain MRI. We did not have specific pathology results in many patients to study their pathological classifications. Patients with dual pathology and also patients with incomplete historical data were excluded from this study.

All patients were submitted to surgical treatment (i.e., anterior temporal lobectomy). They all had to be under the care of an epileptologist at our institution for at least one year, postoperatively. They were followed for up to five years after their surgery (in many patients the data beyond five years of follow-up were missing). Seizure outcome was monitored periodically by office visits (by the treating physician), telephone contacts, and letters (by the nurse practitioners). Postsurgical outcome during follow-up was classified into two groups; sustained seizure freedom or relapse. Aura was not considered as a relapse; only postoperative tonic-clonic seizures and complex partial seizures were considered as relapse. 
Age, gender, race, epilepsy risk factors (e.g., history of febrile seizure, history of tonicclonic seizures, family history of epilepsy, etc.), seizure type(s) and history, EEG findings and MRI findings, date of surgery, date of the first relapse (if any) and date of the last contact with all patients were registered routinely. The type and number of febrile seizures was not registered, so only their presence or absence based on history could be obtained. As a history of febrile seizures is registered based on the history, details are not only often unavailable, but would be unreliable because of the retrospective nature of these reports. Demographic variables and relevant clinical variables were summarized descriptively to characterize the study population. Clinical characteristics and seizure outcomes after epilepsy surgery were compared between patients with or without history of febrile seizures in their childhood. Mantel-Cox, Chi-square, Fisher's Exact tests, and t- test were used for statistical analyses. Time to event analysis was used to produce a Kaplan-Meier estimate of seizure recurrence. A $p$ value less than 0.05 was considered as significant. This study was conducted with the approval by Thomas Jefferson University Institutional Review Board. 


\section{Results}

From 1986 till 2014, 789 patients had anterior temporal lobectomy at our center. Of these, 309 patients (39.2\%) had MTS in their MRI; 262 patients (128 males and 134 females) had MTS-TLE with at least one year of postoperative follow-up and complete data; these were studied. One-hundred and seventy patients (64.9\%) did not have FS in their childhood, while 92 patients $(35.1 \%)$ reported experiencing FS in their childhood. Demographic and clinical characteristics of these two groups of patients are shown and compared in Table 1. Postoperative seizure outcome was not statistically different between these two groups of patients [log rank $($ Mantel-Cox $)=1.667 ; \mathrm{p}=0.19]($ Figure 1$)$. 


\section{Discussion}

Mesial temporal sclerosis probably has different causes. A number of studies showed that febrile seizures are probably one causative factor for the later development of MTS and TLE. However, the association between febrile seizures and TLE probably results from complex interactions between several genetic and environmental factors (7). In this study we tried to investigate whether MTS-TLE with childhood FS is a distinct entity compared to MTS-TLE without FS. Interestingly, demographic and clinical characteristics of these two groups of patients were very similar. In contrast to the previous study by Heuser et al. (6), we did not observe any significant differences with regard to seizure semiology, family history and other characteristics between MTS-TLE patients with or without childhood FS. One probable explanation for these contradictory results is our different methodology, as we selected a very homogenous population of patients with TLE and MTS, while they studied a heterogeneous group of TLE patients including those with MTS. It seems that childhood febrile seizure that has been previously described as a distinguishing feature in the overall group of patients with TLE (6) loses its value when the subgroup of patients with MTS-TLE is examined.

With regard to the relationship between history of childhood febrile seizure and surgery outcome, the data in the literature is more conflicting. In our study, epilepsy surgery outcome was not different between patients with MTS-TLE and drug-resistant seizures, with or without history of childhood febrile seizure in the past. In a previous meta-analysis of predictors of epilepsy surgery outcome, febrile seizures was a strong prognostic indicator of seizure remission (odds ratio 0.48 ; 95\% confidence interval 0.27-0.83) (8). However, this study and most of the studies included in it, investigated a very heterogeneous population of patients with epilepsy (including those with MTS, among other etiologies), who underwent surgery for drug-resistant 
seizures. Our finding that childhood FS is not a predictive factor of surgery outcome in patients with MTS-TLE is similar to those previous studies, which included patients with MTS only (9, 10). It seems that childhood febrile seizure that has been previously described as a predictor of favorable outcome in the overall group of patients receiving temporal lobe resections for drugresistant epilepsy is, in fact, a predictor of MTS and loses its predictive value when the subgroup of patients with MTS is examined (10).

\section{Conclusion}

When mesial temporal sclerosis is the pathological substrate of TLE, clinical manifestations and response to surgical treatment of patients are very similar in patients with history of febrile seizure in their childhood compared with those without such an experience. In other words, when the subgroup of patients with MTS-TLE and drug resistant seizures is examined history of childhood febrile seizure loses its value as a distinguishing factor in characteristics or predictive factor for surgery outcome.

\section{Study limitations}

This was a clinic-based series of patients with medically-refractory MTS-TLE and may not represent the full spectrum of MTS-TLEs. Besides, this study was retrospective and uncontrolled in design. Finally, possible lack of power to detect minor differences between the two groups should also be considered. However, we investigated a larger group of patients including 262 individuals compared with the Heuser's study (207 patients) (6) and we could not reproduce their results. The larger sample size increases the power of the study and decreases the chance of beta (type II) error. Type II errors arise frequently when the sample sizes are too small, 
which was not the case in our study $(11,12)$. For example, for an absolute percentage difference

of $20 \%$ (e.g., $60 \%$ versus $40 \%$ ), which is probably clinically significant for variables such as

outcome, we would have over $85 \%$ power with our sample sizes (two-tailed, alpha $=0.05$ ). For a

difference of $15 \%$ [Heuser et al., observed $15 \%$ (23.5\% vs. $8.5 \%)$ difference in family history of

febrile seizure between their groups (6)], we would have $88 \%$ power. We realize that smaller

effects than these may sometimes still be clinically meaningful, but believe these effect sizes are

reasonable. Therefore, we think that different methodology, as was discussed in the discussion, is

the main reason for different observations between these two studies.

\section{Conflict of interest}

Ali A. Asadi-Pooya, M.D., has no conflict of interest to disclose.

Maromi Nei, M.D., Research: Upsher-Smith Laboratories.

Cyrus Rostami has no conflict of interest to disclose.

Michael R. Sperling, M.D., Consulting: UCB Pharma; Research: contracts with Thomas

Jefferson University, Eisai, UCB Pharma, Sunovion, SK Life Sciences, Marinus, Lundbeck,

Medtronics, Visualase, Accorda, Upsher-Smith, Brain Sentinel, Glaxo, Research support from

DARPA and NIH to Thomas Jefferson University.

Acknowledgment: This was a non-funded study. We thank Edward J. Gracely, PhD., for

statistical assistance. 


\section{References}

1. Wiebe S. Epidemiology of temporal lobe epilepsy. Can J Neurol Sci 2000; 27 Suppl 1: S6-10.

2. Téllez-Zenteno JF, Hernández-Ronquillo L. A review of the epidemiology of temporal lobe epilepsy. Epilepsy Res Treat 2012; 2012: 630853.

3. Kumlien E, Doss RC, Gates JR. Treatment outcome in patients with mesial temporal sclerosis. Seizure 2002; 11(7): 413-417.

4. Provenzale JM, Barboriak DP, VanLandingham K, MacFall J, Delong D, Lewis DV. Hippocampal MRI signal hyperintensity after febrile status epilepticus is predictive of subsequent mesial temporal sclerosis. Am J Roentgenol 2008; 190(4): 976-983.

5. Fernández G, Effenberger O, Vinz B, et al. Hippocampal malformation as a cause of familial febrile convulsions and subsequent hippocampal sclerosis. Neurology 1998; 50(4): 909-917.

6. Heuser K, Cvancarova M, Gjerstad L, Taubøll E. Is Temporal lobe epilepsy with childhood febrile seizures a distinctive entity? A comparative study. Seizure 2011; 20(2): $163-166$.

7. Cendes F. Febrile seizures and mesial temporal sclerosis. Curr Opin Neurol 2004; 17(2): $161-164$.

8. Tonini C, Beghi E, Berg AT, et al. Predictors of epilepsy surgery outcome: a metaanalysis. Epilepsy Res 2004; 62(1): 75-87.

9. Hennessy MJ, Elwes RD, Rabe-Hesketh S, Binnie CD, Polkey CE. Prognostic factors in the surgical treatment of medically intractable epilepsy associated with mesial temporal sclerosis. Acta Neurol Scand 2001; 103(6): 344-350. 
10. Hardy SG, Miller JW, Holmes MD, et al. Factors predicting outcome of surgery for intractable epilepsy with pathologically verified mesial temporal sclerosis. Epilepsia 2003; 44(4): 565-568.

11. https://www.easycalculation.com/statistics/learn-beta-error.php/12.11.2015

12. lap.umd.edu/psyc200/handouts/PSYC200_0803.pdf/12.11.2015 
Figure 1 legend. Postoperative outcome was not statistically different between these two groups of patients $(\mathrm{p}=0.19)$. Numbers of the patients at risk at each time point for the two curves are shown below.

\begin{tabular}{|l|c|c|c|c|c|}
\hline & \multicolumn{5}{|c|}{ Number of the remaining cases at each time point (year) } \\
\hline Time point (year) & 0 & 1 & 2 & 3 & 4 \\
\hline Patients without & 170 & 96 & 82 & 65 & 52 \\
febrile seizures & & & 51 & 42 & 33 \\
\hline Patients with & 92 & 62 & & & \\
\hline
\end{tabular}

